\title{
Álvaro López Toro: aportes a la demografía y a los estudios sobre la población ${ }^{1}$
}

\section{Álvaro López Toro: Contributions in demography and population studies}

\section{Introducción}

Quiero iniciar este comentario manifestando la gran satisfacción que siento por la invitación de la Academia Colombiana de Ciencias Económicas para reflexionar sobre la vida y obra de uno de los científicos colombianos más reconocidos en el mundo por su invaluable contribución al desarrollo de la demografía matemática, los estudios sobre la estructura y dinámica de las poblaciones humanas y el análisis de las interrelaciones entre el cambio demográfico y el desarrollo económico y social en los países latinoamericanos. Debo manifestar que esta tarea no ha sido fácil por las características personales, la vastedad y profundidad de la obra de Álvaro López Toro.

Por fortuna, sus más distinguidos admiradores, Rodolfo Heredia Benítez y Luciano Mora Osejo, con el respaldo de Francisco Ortega, entonces gerente del Banco de la República, emprendieron la compleja labor de indagar a sus familiares sobre su vida y a recopilar casi la totalidad de la obra en el libro titulado: Álvaro López Toro. Ensayo sobre Demografía y Economía, publicado en 1991 por el Banco de la República. Sin duda, este libro simplificó considerablemente mi trabajo de investigación, pero no evitó el problema de tratar de sintetizar en las pocas páginas de este texto, su obra de enormes dimensiones, a pesar de su desaparición temprana. Pido, entonces, indulgencia por la omisión de algunos aspectos que, posiblemente, en criterio de los compiladores, se deberían haber incluido en este apretado resumen. Por lo tanto, los errores y las omisiones son responsabilidad exclusiva mía y no comprometen para nada a sus avezados biógrafos.

\section{Semblanza personal de Álvaro López Toro}

Álvaro López Toro nació en Medellín en 1926. Cursó estudios primarios en el Ateneo antioqueño y secundarios en el Liceo de la Universidad de Antioquia, del cual se graduó como Bachiller en Filosofía y Letras en 1943. Al año siguiente ingresó a la Escuela Nacional de Minas para adelantar estudios de ingeniería, debido a que no se impartía la carrera de matemáticas en esa época en la ciudad de Medellín.

Al terminar los estudios profesionales en 1948, obtuvo una beca para estudiar una especialización en Estadística en la Facultad de Ciencias de la Universidad de París, durante 1949 y 1950. A su regreso al país, contrajo matrimonio con Ángela Mejía, unión de la cual nacieron dos hijos, Adelaida y Alejandro.

Durante los años siguientes se desempeñó como asistente de investigación del Banco de la

1 Intervención presentada en la tertulia de la Academia Colombiana de Ciencias Económicas el 11 de septiembre del 2016 en Bogotá.

2 Postdoctor con estancia posdoctoral en la University of North Carolina. Ph.D. en Economía de Poblacion y Desarrollo Económico de la University of North Carolina, Estados Unidos. Economista de la Universidad del Valle, Cali, Colombia. Rector de la Fundación Universitaria Lumen Gentium, Cali, Colombia. Miembro correspondiente de la Academia Colombiana de Ciencias Económicas. Investigador cied, Universidad Autónoma de Occidente, Cali, Colombia. Correo electrónico: rectoria@unicatolica.edu.co 
República, investigador en economía y actuaria, y director de investigaciones en la Asociación Nacional de Empresarios de Colombia (Andi) en Medellín (Mejía, Mora y Ruiz, en Heredia y Mora, 1991, pp.17-18).

Fue designado consejero económico de la Embajada de Colombia en Washington en 1958. Esta designación marcó un hito importante en la carrera científica de Álvaro López, puesto que, aunque siendo catedrático de la Escuela de Ingeniería, ya había publicado su primer trabajo sobre demografía en 1957, que se tituló "Dos aplicaciones demográficas del Diagrama de Lexis-Vincent", tuvo un encuentro casual en Washington con Frank Lorimer, distinguido demógrafo norteamericano, quien, al ver su interés y aptitud para los estudios demográficos, lo recomendó para que fuera admitido a la Universidad de Princeton, a la cual ingresó en 1958, becado inicialmente por la oEA y posteriormente por el Population Council.

En esa universidad tuvo la oportunidad de conocer y trabajar con un grupo de economistas-demógrafos, liderado por Ansley Coale, director de la Oficina de Investigaciones en Población que se dedicaba a realizar desarrollos en el campo de la demografía matemática, particularmente en la validación de la teoría de las poblaciones estables y sus implicaciones demográficas. Sus aptitudes excepcionales para las matemáticas y en particular, su dominio del álgebra lineal, le permitieron validar matemáticamente los teoremas de ergodicidad débil y fuerte -propuestos anteriormente por el profesor Coale- que habían sido comprobados empíricamente mediante proyecciones de largo plazo de poblaciones específicas, mas no existía una demostración matemática de ellos. El profesor Coale le sugirió hacer la demostración matemática de estos teoremas como tema de tesis doctoral y le prometió graduarlo inmediatamente si lo lograba, López Toro solo necesitó unos pocos meses para hacerlo. Su tesis doctoral titulada: "Problems in Stable Population Theory", presentada dos años después de hacer su ingreso a la Universidad, le permitieron obtener su doctorado en un tiempo récord. Este trabajo es hoy en día, referencia obligada para todo aquel que aspire a trabajar temas de demografía matemática en el mundo y, en mi opinión, constituye uno de los aportes más significativos y pieza fundamental para el desarrollo de la demografía en el siglo xx. (Mejía, Mora y Ruiz, citados en Heredia y Mora, 1991, pp.18-19).

Cabe mencionar una anécdota personal que muestra la dimensión de lo que López Toro llevó a cabo en su trabajo doctoral. En mis estudios doctorales, realizados en la Universidad de Carolina del Norte en Chapel Hill, uno de los requisitos para grado era cursar dos semestres de demografía matemática avanzada en la prestigiosa Escuela de Salud Pública de esa universidad. En el primer día de clase mi profesor -de origen hindúpreguntó por las nacionalidades de los estudiantes y al escuchar mi origen, con gran sorpresa me dijo: Ah, usted es colombiano, la tierra de Álvaro López Toro, espero que sea tan buen estudiante como él. Por supuesto, ya pueden imaginarse lo que me tocó estudiar en esos cursos -plagados de referencias a los trabajos de López Toro- para obtener las mejores calificaciones y no defraudar así al profesor. Obtuve excelentes notas en los dos cursos, un logro modesto comparado con los de López en Princeton. Sin embargo, en la última sesión de clase, nuevamente el profesor hizo el siguiente comentario: Qué tienen estos colombianos que son tan buenos demógrafos. Solo atiné a decir para mis adentros: Gracias Álvaro López por plantearme semejante desafío. Cómo valoré en ese momento la excelente formación matemática que había recibido años atrás en la Universidad del Valle.

Al terminar sus estudios doctorales, López Toro regresó a Colombia en 1960 y decidió combinar la consultoría sobre temas económicos con la cátedra en la Universidad de los Andes. En 1965 regresó a la Universidad de Princeton como profesor visitante de la Escuela de Postgrado, en la Woodrow Wilson School of Economics y a la Universidad de Pensilvania, en la que lideró cátedras sobre temas de población y economía. En este período, con el 
soporte de la Oficina de Investigaciones en Población de la Universidad de Princeton, escribió los artículos "Propiedades asintóticas de una distribución por edades de una población humana bajo una función continua de maternidad neta", "Notas sobre problemas de fecundidad en una comunidad semi-urbana de Colombia", "Patrones de nupcialidad en Bogotá" e inició a trabajar en un libro: Migración y cambio social en Antioquia durante el siglo XIX (Mejía, Mora y Ruiz, citados en Heredia y Mora, 1991, pp.19-20).

En 1967 la Universidad de los Andes -interesada en vincularlo al país- lo llamó para que dirigiera el Programa de Demografía del Centro de Estudios sobre Desarrollo Económico (Cede), allí López Toro dedicó todos sus esfuerzos a fundar una escuela en la cual los aspirantes a demógrafos tuvieran la oportunidad de formarse con rigor científico en demografía y en estudios sobre población. Impartió el curso Demografía General para Economistas, escribió varios artículos sobre demografía y el libro Análisis demográfico de los censos colombianos 1951-1964, un trabajo pionero en este campo en el país y que solo él podía realizar, gracias a su profundo conocimiento de la teoría sobre poblaciones estables y sus aplicaciones. Ejerció dicha labor hasta 1971, fecha en la cual se cerró el Programa, dejó como legado para el país, una generación de jóvenes demógrafos que se beneficiaron de sus conocimientos y continuaron la tarea iniciada por él. Como colofón a su trabajo, en 1971 Colciencias le otorgó el premio Caldas de Ciencia, máxima distinción otorgada a científicos colombianos distinguidos.

En agosto de 1971 se retiró de la Universidad de los Andes y se vinculó como asesor económico del Banco de la República, cargo en el cual permaneció hasta el final de sus días, en enero de 1972.

Como lo expresaron los compiladores Heredia y Mora (1991):

Esta biografía quedaría incompleta si no se hiciera referencia a los criterios que guiaron personalmente su trabajo científico y que constituyen los rasgos principales de su obra, la metodología que le permitió alcanzar un altísimo grado de disciplina y rigurosidad científica. Estos criterios siguieron las siguientes pautas: antes de abordar un tema o simultáneamente con su desarrollo, se presenta exhaustivamente el "estado de la cuestión", los orígenes del problema, las variantes, los resultados positivos, las cuestiones abiertas a la investigación; rigor conceptual y metodológico a lo largo de todo el tratamiento de un problema, aún a riesgo de mostrarse extravagante y repetitivo, brillante capacidad para explorar en una dirección imprevista ideas y métodos que estaban sobre la arena de la investigación; notable precisión y propiedad en utilizar los métodos matemáticos en cuyo desarrollo se mantenía asombrosamente actualizado; sentido claro de los peligros implícitos en las conclusiones tratadas vagamente; claro convencimiento de que el objetivo final de los rigorismos teóricos es la apertura de nuevos campos de aplicación y la construcción de instrumentos operativos eficaces. (Mejía, Mora y Ruiz, en Heredia y Mora, 1991, p. 20)

\section{El aporte de Álvaro López Toro a la demografía y a los estudios sobre la población y el desarrollo}

Según la tipificación de Heredia y Mora (1991) y para efectos del análisis, clasifico su obra en tres categorías de aportes, curiosamente escritos casi en su totalidad en el mismo orden: i) trabajos en demografía matemática, piedra angular de su producción intelectual, ii) estudios de población y iii) estudios sobre población y desarrollo.

\section{i. Aportes a la demografía matemática}

Como se indicó en la sección anterior, López Toro escribió por primer vez sobre demografía matemática a su regreso de Francia, se tituló "Dos aplicaciones demográficas del diagrama de LexisVincent" (López Toro, 1957). En este trabajo López Toro presentó de manera rigurosa la relación existente entre el paso del tiempo y la edad de las personas, fundamental para el seguimiento de cohortes de población a partir de datos de corte transversal. Posteriormente, mostró cómo usar el 
diagrama para retropolar una población a la fecha $1^{\circ}$ de enero de un año censal, con el propósito de calcular la probabilidad de muerte al inicio del año y usarlas para la construcción de tablas de vida o para hacer análisis de la validez de los datos censales. Su exposición detallada del procedimiento matemático y de los ejemplos de aplicación marcó el inicio de una manera para abordar temas demográficos que lo acompañó durante el resto de su vida.

Su segundo trabajo en este campo es, sin duda, su obra magistral, la tesis doctoral titulada Problems in stable population theory (Problemas de la teoría de las poblaciones estables) (López Toro, 1961), en la que hace un despliegue total de sus conocimientos y destrezas matemáticas para definir el estado del arte sobre el concepto de las poblaciones estables. Parte de los planteamientos iniciales de Lotka (1939, 1948), Coale (1957) y los desarrollos matemáticos de Hajnal (1956, 1958), hace gala de su dominio de las propiedades asintóticas del algebra lineal para demostrar que, en el largo plazo, una población cerrada (sin migración) sometida a regímenes constantes de fecundidad y mortalidad, olvida su pasado y converge a una estructura de edad constante denominada población estable, conocido como el teorema de la ergodicidad débil en demografía.

Posteriormente, también logra demostrar matemáticamente cómo dos poblaciones con características iniciales diferentes, sometidas a regímenes similares de fecundidad y mortalidad, convergen en el largo plazo a la misma estructura de edad constante, conocido como el teorema de la ergodicidad fuerte. En la parte final de su trabajo hace uso de datos colombianos para mostrar la aplicabilidad de los modelos de poblaciones estables para estimar los parámetros demográficos (fecundidad, mortalidad, tasa de crecimiento y estructura de la población por edad) con datos censales deficientes. Las demostraciones de estos teoremas que López Toro realiza en su tesis doctoral son hoy en día un referente obligado para los estudiosos de la demografía matemática en muchos países alrededor del mundo. Paradójicamente, su nombre ha sido más referenciado en el exterior que en Colombia.

Para darle continuidad al trabajo que consolidó en su tesis doctoral, López Toro publicó un artículo en 1967 en la prestigiosa revista Demography, titulado "Propiedades asintóticas de una distribución etaria de una población humana bajo una función continua de maternidad neta" (López Toro, 1967a), en el cual expone la demostración matemática de la validez de los teoremas de ergodicidad débil y fuerte en el caso continuo, en esta ocasión emplea el cálculo diferencial. De esta forma, López Toro completó la tarea que se había impuesto desde el inicio de su trabajo doctoral en Princeton. En mi opinión, solo una persona con el conocimiento y disciplina de Álvaro López Toro podría realizar las demostraciones matemáticas de estos teoremas, fundamentales para el desarrollo de la demografía como ciencia.

\section{ii. Aportes a los estudios de la población}

La segunda fase de los trabajos de López Toro se centra en el análisis de las interrelaciones entre las variables demográficas de fecundidad, mortalidad, migración y nupcialidad, y un conjunto de variables económicas y sociales, algo conocido en la literatura como estudios de población; para ellos recurrió a información de diferentes fuentes disponibles en Colombia y al análisis de problemas en los censos, aplicó su profundo conocimiento de la teoría de las poblaciones estables y sus posibilidades de aplicación para detectar errores de subestimación censal.

En el primero de estos estudios, "Notas sobre problemas de fecundidad en una comunidad semiurbana de Colombia" (1967b), López Toro utilizó información disponible sobre la vida reproductiva de cien mujeres en el municipio de Candelaria (Valle del Cauca) para estudiar a profundidad el comportamiento reproductivo de dos grupos, y así 
determinar si existía diferencia en la relación entre fecundidad (número de hijos) y la frecuencia de relaciones sexuales (fecundabilidad) entre las mujeres casadas y las no casadas. La conclusión fue que no había tal diferencia. López Toro advirtió sobre el peligro de aceptar como válida la creencia común de ese entonces de que una alta fecundidad estaba asociada con una mayor frecuencia de relaciones sexuales. Su estudio mostraba lo contrario, algo que hoy en día es evidente por el papel que juegan los métodos anticonceptivos en la diferenciación entre la fecundabilidad (el potencial) y la fecundidad (hijos efectivamente engendrados).

El segundo trabajo en esta categoría se denomina Patrones de nupcialidad en Bogotá, publicado en 1968 por la Oficina de Investigaciones sobre Población de la Universidad de Princeton (López Toro, 1968b), en este aprovechó la información disponible en la Encuesta de Fecundidad del Celade, realizada en Bogotá en 1964 para estimar algunos índices de nupcialidad, evaluar los efectos de la migración sobre los patrones de nupcialidad e identificar tendencias en la edad al matrimonio entre cohortes sucesivas. Hasta donde llega mi conocimiento, este estudio es el primer intento de un demógrafo de estudiar la nupcialidad y sus determinantes en Colombia.

En el tercero de los trabajos de esta categoría -inédito en este caso- "Notas sobre los fenómenos migratorios del Valle del Cauca" de 1969, López Toro utilizó información del Censo de Población de 1964 y la tabuló con los datos sobre migración intermunicipal e interdepartamental para el Valle del Cauca que había publicado la Universidad del Valle, analizó en detalle los flujos migratorios entre los municipios al interior del Departamento y de este con los demás departamentos del país (López Toro, 1969a.). Comprobó la intensidad alta de las corrientes migratorias existentes en los municipios clasificados en categorías según su tamaño; destacó el hecho de que estas corrientes son de todo tipo: rural-urbana, rural-rural y urbana-urbana, producto de los grandes desplazamientos que iniciaban en Colombia como consecuencia de la violencia política. En el caso de las migraciones interdepartamentales, López Toro percibió una tendencia a la compensación de los flujos de emigrantes con los inmigrantes, y evidenció nuevamente una gran movilidad espacial de la población colombiana de la época.

Tres de los trabajos incluidos en esta categoría son ejercicios hechos por López Toro al aplicar su dominio sobre las propiedades derivadas de la teoría de las poblaciones estables para: i) estimar la fecundidad y la mortalidad en Colombia con la información de los censos de 1938, 1951 y 1964, en el trabajo "Nuevas técnicas para estimar la fecundidad y la mortalidad: el caso colombiano", publicado en 1968 (López Toro, 1968a); ii) estimar parámetros demográficos a partir de datos censales con errores de cobertura en el trabajo "Algunas posibilidades de estimar medidas demográficas a partir de datos censales con errores de cobertura", ponencia presentada a la Conferencia General de la Unión Internacional para el Estudio Científico de la Población, realizada en Londres en 1969 (López Toro, 1969b); y iii) hacer un exhaustivo análisis de dos censos colombianos en el trabajo: "Análisis demográfico de los censos colombianos 1951 y 1964" -publicado por la Universidad de los Andes en 1968-, en términos de su cobertura, errores de omisión en la enumeración en algunas edades y estimación de la fecundidad, la mortalidad y la tasa de crecimiento de la población (López Toro, 1968c), para ello empleó un promedio de los modelos oeste y sur de las tablas de poblaciones estables elaboradas por Coale y Demeny (1966). Evidenció en este trabajo el gran subregistro que presentaban la cifras sobre nacimientos y defunciones en las estadísticas vitales, algo que aún hoy no ha sido posible corregir por completo.

Finalmente, se incluye en esta categoría el trabajo que realizó junto con Roberto Junguito, Álvaro Reyes y Diego Pizano: "Análisis de la estructura y evolución de la fuerza de trabajo colombiana 1938-1951-1964 y proyecciones de población económicamente activa 1965-1985", en el que analizan los datos sobre la oferta laboral colombiana en los 
tres censos para obtener tendencias en su comportamiento en el período 1938-1964, para luego utilizarlas en una proyección de la población económicamente activa de Colombia hasta 1985, sin duda, uno de los primeros ejercicios hechos en el país sobre el tema (Junguito et al., 1970).

\section{iii. Aportes a los estudios sobre población y desarrollo}

En esta sección se aborda un conjunto de trabajos de López Toro enfocados en el análisis de las interrelaciones entre la dinámica demográfica y el desarrollo económico y social en los países en vías de desarrollo. Algunos de ellos tienen particular significado para mí, puesto que fueron fuente de inspiración para mi trabajo de tesis doctoral y, posteriormente, para la construcción del modelo Colombia 2000 en el CEDE de la Universidad de los Andes.

Inicio la síntesis con el trabajo "Factores demográficos en el desarrollo económico de América Latina", ponencia presentada en la Conferencia sobre Problemas Demográficos Latinoamericanos, llevada a cabo en la Universidad de Cornell en 1968 (López Toro, 1968d), en este López Toro hace un recorrido por los principales planteamientos de diversos autores sobre el impacto de los cambios en la fecundidad, mortalidad y migración sobre el desarrollo económico de los países latinoamericanos. Trata a profundidad temas como la presión ejercida por la población sobre la tierra y los demás recursos naturales, la relación entre tamaño de población y mercado potencial, los beneficios y costos económicos del control natal, el impacto de altas tasas de natalidad sobre la inversión y el crecimiento económico y los problemas estructurales que limitan las posibilidades del desarrollo económico en los países latinoamericanos.

El análisis del impacto de la dinámica poblacional sobre el desarrollo económico y social fue objeto de estudio de López Toro desde su llegada a la Universidad de Princeton. De esta época data un trabajo desarrollado con Paul Demeny en 1959, "Construcción de modelos generales de desarrollo económico y social", en el cual plantearon un modelo macroeconómico de corte neoclásico (Solow, 1956) para estudiar el impacto de los cambios en la fecundidad sobre el ingreso por habitante. La introducción de la dinámica del mercado laboral en el modelo les permitió concluir que en efecto, hay un impacto positivo de la reducción de la fecundidad sobre el ingreso por habitante, siempre y cuando se dé una situación de pleno empleo en el mercado laboral. En la segunda parte del trabajo utilizaron un modelo del tipo Harrod (1939) o Domar (1946) para evaluar el impacto del ahorro generado por la reducción en la fecundidad sobre la tasa de crecimiento de la producción, bajo el supuesto de que todo lo que se ahorra se invierte. En la parte final, desarrollaron un modelo dual que hacía una distinción entre el sector agrícola y el no agrícola para tener en cuenta las diferencias existentes entre ellos en términos demográficos y económicos, algo imposible de captar con el modelo de un solo sector. Concluyeron que el impacto de la reducción de la fecundidad sobre el ingreso por habitante no necesariamente es positivo, ya que depende de las condiciones estructurales de la economía, particularmente del mercado laboral y de la materialización en inversión adicional del ahorro generado por la menor fecundidad (Demeny y López Toro, 1959).

En un segundo trabajo sobre el tema, "Algunas ideas referentes al concepto de sobrecarga demográfica" (1968e), el autor utiliza el modelo de Swan (1956) con algunas modificaciones para analizar el impacto del cambio tecnológico sobre la producción bajo rendimientos decrecientes y un alto crecimiento poblacional. Al final concluye que la capacidad del cambio tecnológico para compensar el efecto de la alta tasa de crecimiento poblacional depende críticamente de la función de bienestar a maximizar en una sociedad y de las condiciones de empleo de la población (López Toro, 1968e).

En el trabajo "Inversiones demográficas en una economía abierta” (López Toro, 1969c), López Toro 
hace una ampliación del modelo desarrollado por Enke (1966) para evaluar los costos y beneficios de los programas de planificación familiar versus la inversión directa en otros proyectos de desarrollo en una economía cerrada, para incorporar el hecho real de que existe ayuda externa para estos propósitos, algo que modifica la restricción del ahorro interno en el análisis. Concluye que, en efecto, el costo de oportunidad de los recursos externos para el control natal es diferente en el tiempo y depende de la existencia o no de una brecha externa en la economía (López Toro, 1969c).

Algunas de las sugerencias hechas por López Toro en estos tres trabajos fueron posteriormente retomadas por un grupo de investigadores de la Corporación Centro Regional de Población para la construcción del modelo de simulación Seres (Sistema para el Estudio de la Relaciones Económicas, Sociales y Demográficas) cuyo propósito recae en evaluar el impacto del cambio en variables demográficas sobre la economía, el sector público y el bienestar de los colombianos (CCRP, 1975; 1977).

Los trabajos anteriores analizan el impacto del cambio demográfico sobre el desarrollo económico bajo el supuesto de que la causalidad es en esa dirección, pero ignoran la existencia de la causalidad en el otro sentido: del desarrollo económico y social hacia la dinámica poblacional. Con estos trabajos como punto de partida, en 1978 tuve la oportunidad de liderar un equipo de investigadores en el CEDE de la Universidad de los Andes para la construcción de un modelo de simulación macroeconómica conocido como Colombia 2000, en este se eliminan los supuestos exógenos sobre el comportamiento de la fecundidad, mortalidad y migración y se estiman endógenamente en función del comportamiento de variables económicas y sociales para crear así, un sistema económicodemográfico totalmente autodeterminado para Colombia (Banguero, 1980b; 1982).

Un trabajo que merece una mención destacada en este resumen es "Migración y marginalidad urbana en países desarrollados" (López Toro, 1970b), en el cual López Toro hace gala de su conocimiento y agudeza matemática para encontrar un error de factorización en el modelo planteado por Todaro (1969) para explicar la decisión de migrar del campo a la ciudad en función de la diferencia relativa entre los ingresos esperados y la probabilidad de encontrar empleo en el sector urbano. López Toro no solo corrigió el error, sino que mostró, de manera muy profesional y académica, que las conclusiones obtenidas por Todaro son exactamente las contrarias a las que él obtuvo con el modelo corregido. Posteriormente sugirió la endogenización del modelo, dado que la relación de causalidad entre migración y el diferencial relativo de ingresos y el empleo se da en ambas direcciones. Sapoznikow retomó estas ideas básicas planteadas por López Toro para construir un modelo de crecimiento económico con tres sectores en el cual se incorporan de manera explícita, funciones de migración similares a las planteadas por López Toro en el artículo (Sapoznikow, 1976a, 1976b).

En el trabajo "Migración y cambio social en Antioquia durante el siglo xIx" (1976), López Toro analiza de manera crítica las interpretaciones de Parsons (1950), Havens (1966), Hagen (1962) y Safford (1967) sobre el impacto de los flujos migratorios hacia esta región en la época colonial en la dinámica económica y social de Antioquia en el siglo xIx. Aunque el estudio es de naturaleza histórica, refleja la capacidad de López Toro para analizar críticamente publicaciones de autores provenientes de disciplinas tan diferentes como la geografía, la psicología y la sociología.

Finalmente, en esta categoría se incluye el trabajo "Temas sobre población y desarrollo económico en América Latina” (1970a), en el cual López Toro presenta el estado del arte de la investigación en América latina en lo referente a: i) la presión demográfica sobre los recursos naturales y de capital; ii) los aspectos económicos de la fecundidad; iii) la mortalidad, morbilidad y desarrollo económico y social; y iv) la tasa de crecimiento demográfico; también señala temas prioritarios para futuras investigaciones. 
Sus reflexiones sobre los aspectos económicos de la fecundidad fueron fuente de inspiración para el tema de mi tesis doctoral, en la cual hago una generalización de la teoría del valor del hijo para explicar el tamaño de la familia en países en desarrollo, incluyo en un solo modelo los costos y los beneficios esperados de los hijos por sus padres, ya que, a diferencia de los países desarrollados en los que el hijo es visto solo como un costo para sus padres, en muchas familias de países en desarrollo el hijo se percibe más bien como un beneficio que como un costo, por los aportes monetarios y no monetarios que hace a sus padres a lo largo de su ciclo de vida. En familias de muy bajos recursos los hijos son el eje de una estrategia de supervivencia familiar y de mano de obra gratuita y por lo tanto, tener familias grandes es una decisión tan racional en estos casos como la de no tenerlos en el caso contrario. La validación de la relación existente entre el valor del hijo y la fecundidad, ya esbozada por López Toro en su estudio (1970a) que se vale de datos de la cuarta encuesta de ingresos y gastos realizada en Colombia por el Dane en 1970, me permitió culminar exitosamente mis estudios doctorales en la Universidad de Carolina del Norte, Chapel Hill, a finales de 1977 (Banguero, 1979, 1980a).

Al terminar la revisión que he hecho de su obra científica, no me cabe la menor duda de que Álvaro López Toro marcó un hito en el desarrollo de la demografía y de los estudios de población en Colombia y en el mundo y que constituye un ejemplo a seguir para personas de esta y futuras generaciones de colombianos. Sus estudios no solo abordaron los aspectos teóricos, López Toro tuvo especial cuidado en aplicar sus conocimientos de manera sistemática al análisis de problemas concretos de la realidad colombiana y latinoamericana.

\section{Reflexión final}

Luego de hacer un recorrido por su obra, se percibe la preocupación de López Toro por dos grandes temas: i) la deficiente calidad de los datos demográficos, en particular de los censos de población y de los registros vitales; y ii) la necesidad de formar demógrafos tanto en Colombia como en toda América Latina, con las competencias requeridas para emular a los homólogos de los países más desarrollados. Sin duda, su obsesión fue la creación de una escuela de demógrafos para Colombia y América Latina, tarea que inició en la Universidad de los Andes, pero que desafortunadamente se frustró muy pronto.

Quisiera proponer que, bajo el liderazgo de la Academia Colombiana de Ciencias Económicas, como consecuencia de esta tertulia y en homenaje a la vida y obra de Álvaro López Toro se impulsen dos iniciativas para intentar dar respuesta, así sea tardía, a estas dos inquietudes:

i. La presentación al Congreso de la República de la Ley de Población, para regular todo lo referente a la información sociodemográfica del país, en la cual se definan los lineamientos para: i) la realización de un Censo de Población y Vivienda cada diez años en una fecha determinada, ojalá en un año terminado en cero o cinco, recomendación de Naciones Unidas, al igual que las características del mismo en términos de estructura y la metodología para su planeación, elaboración, procesamiento y divulgación, bajo las recomendaciones de las Naciones Unidas al respecto, de tal forma que su realización deje de ser objeto de una decisión política del gobernante de turno y se haga con regularidad en una fecha y año prestablecidos, bajo el liderazgo del DANE, con la colaboración de los entes territoriales en el proceso de recolección de la información, como corresponde en un país descentralizado. Esta es una práctica común en los países más desarrollados, por ser la población y su localización el eje fundamental de cualquier proceso de planificación; ii) reglamentar en esta ley todo lo relacionado con el proceso de 
recolección, procesamiento y divulgación de las estadísticas vitales, con el propósito de mejorar su cobertura y calidad; y iii) la realización anual con representatividad nacional de una encuesta de calidad de vida de la población colombiana, con el propósito de obtener la información requerida para crear un sistema de cuentas sociales - similar al sistema de cuentas económicas nacionales- que permita hacer monitoreo permanente del progreso de los indicadores sociales en materia de nutrición y salud, vivienda y servicios básicos, educación, recreación y deporte, empleo y seguridad social, libertad, seguridad, pertenencia y afectividad en la población que reside en el país, departamentos y municipalidades (Banguero, 2004).

ii. La creación en alguna de las universidades de Colombia de una Escuela de Estudios en Demografía, Población y Desarrollo, responsable no solo de la formación de profesionales en este campo a nivel de maestría y doctorado, sino de investigación para abordar, ya no los temas de la fecundidad y la mortalidad relevantes en la época de la explosión demográfica causada por la alta fecundidad y la caída de la mortalidad en el siglo pasado, sino aquellos de la migración y envejecimiento, por ejemplo, desplazamiento forzado, atención en salud y financiamiento de las pensiones de los adultos mayores. Intentos personales hechos con algunas universidades para lograr este objetivo en el pasado no tuvieron la acogida esperada, a pesar de su evidente necesidad. Hoy más que nunca, Colombia y Latinoamérica necesitan demógrafos competentes para abordar los nuevos desafíos en materia de población y desarrollo en nuestros países y pienso que las universidades no pueden seguir siendo indiferentes a este requerimiento, porque el costo social de no hacerlo es demasiado grande.

Gracias.

\section{Referencias}

Banguero, H. (1979). El costo monetario neto de un hijo en Colombia. Documentos CEDE, (55).

Banguero, H. (1980a). El tamaño de la familia colombiana: sus determinantes económicos y sociales. Documentos CEDE, (60).

Banguero, H. (1980b). Colombia 2000: un marco para la planificación de la población, el empleo, el crecimiento, la distribución del ingreso y las necesidades humanas esenciales. Documentos CEDE, (67).

Banguero, H. (ed.) (1982). Colombia 2000. Estrategias de desarrollo para satisfacer las necesidades bumanas esenciales. Editorial Dintel.

Banguero, H. (2004). Fuentes de información demográfica. En El análisis de la población. Conceptos, métodos y aplicaciones (cap. 3, pp. 67 - 93) Editorial Universidad Autónoma de Occidente.

ccrp (1975). Modelo Seres [Sistema para el Estudio de las Relaciones Económicas, Sociales y demográficas]. Descripción técnica. Documento Técnico No. 5 (vol. 1). Bogotá: Corporación Centro Regional de Población.

ccrp (1977). Modelo Seres. Estructura y usos. Monografía No. 3. Bogotá: Corporación Centro Regional de Población.

Coale, A. (1957). How the age distribution of a human population is determined. Cold Spring Harbor Symposia on Quantitative Biology, 22, 83-89. http://dx.doi.org/10.1101/SQB.1957.022.01.010

Coale, A., y Demeny, P. (1966). Regional Model Life Tables and Stable Populations. Princeton University Press.

Demeny, P., y López Toro, Á. (1959). Construction of general models of economic and social 
development [Mimeo]. Presentado en Seminario sobre la evaluación y utilización de datos censales de población en Latinoamérica. Santiago de Chile.

Domar, E. D. (1946, abril). Capital expansion, rate of growth and employment. Econometrica, 14(2), 137-147. Recuperado de https://laprimaradice. myblog.it/media/00/00/2491562877.pdf

Enke, S. (1966, marzo). The economic aspects of slowing population growth. Economic Journal, 76(301), 44-56. https://doi.org/10.2307/2229036

Hagen, E. (1962). On the theory of social change. The Dorsey Press Inc.

Hajnal, J. (1956). The ergodic properties of nonhomogeneous finite Markov chains. Proceedings of the Cambridge Philosophical Society, 52. Part. I.

Hajnal, J. (1958). Weak ergodicity in nonhomogeneous Markov chains. Proccedings of the Cambridge Philosophical Society, 54. Part. II.

Havens, E. (1966). Támesis, estructura y cambio. Ediciones Tercer Mundo.

Harrod, R. F. (1939, marzo). An essay in dynamic theory. The Economic Journal, 49(193), 14-33. Recuperado de http://piketty.pse.ens.fr/files/ Harrod1939.pdf

Heredia, R., y Mora Osejo, L. (comps). (1991). Álvaro López Toro. Ensayos sobre demografía y economía. Editorial Banco de la República.

Junguito, R., López Toro, Á., Reyes, Á., y Pizano, D. (1970). Análisis de la estructura y evolución de la fuerza de trabajo colombiana 1938 - 1951 - 1964 y proyecciones de población económicamente activa 1965 - 1985. Universidad de los Andes.

López Toro, Á. (1957). Dos aplicaciones demográficas del diagrama de Lexis- Vincent. Estadística, 15(54).

López Toro, Á. (1961). Problems in stable population theory. Office of Population Research. Princeton University.

López Toro, Á. (1967a). Asymptotic properties of a human age distribution under a continuous net maternity function. Demography, 4 (2), 680-687. https://doi.org/10.2307/2060308

López Toro, Á. (1967b). Some notes on fertility problems in a Colombian semi- urban community. Demography, 4 (2), 453-463.

López Toro, Á. (1968a). New techniques to estimate fertility and mortality: the case of Colombia. Memorial Milbank Fund Quarterty, xlvi(3),

López Toro, Á. (1968b). Nuptiality patterns in Bogotá [Mimeo]. Princeton Office of Population Research.

López Toro, Á. (1968c). Análisis demográfico de los censos colombianos 1951 y 1964. Ediciones Universidad de los Andes.

López Toro, Á. (1968d, enero-febrero). Factores demográficos en el desarrollo económico de América Latina. Razón y Fábula, (5),

López Toro, Á. (1968e). Algunas ideas referentes al concepto de sobrecarga demográfica [Mimeo]. Revista de Planeación y Desarrollo.

López Toro, Á. (1969a). Notas sobre los fenómenos migratorios del Valle del Cauca [Mimeo]. Boletín Mensual de Estadistica, (216).

López Toro, Á. (1969b). Algunas posibilidades de estimar medidas demográficas a partir de datos censales con errores de cobertura. Presentado en Conferencia General de la International Union for the Scientific Study of Population. London.

López Toro, Á. (1969c). Inversiones demográficas en una economía abierta [Mimeo]. CEDE. Revista de Planeación y Desarrollo.

López Toro, Á. (1970a). Temas sobre población y desarrollo en América Latina. Presentado en Conferencia Regional Latinoamericana de Población. Biblioboletín (5), CEDE.

López Toro, Á. (1970b). Migración y marginalidad urbana en paises subdesarrollados [mimeo]. Cede. Universidad de los Andes.

López Toro, Á. (1976). Migración y cambio social en Antioquia durante el siglo XIX. Monografía No. 25. CEDE. Facultad de Economía. Universidad de los Andes. 
Lotka, A. (1939). Teoría analítica de las asociaciones biológicas. Parte xii: análisis demográfico con particular aplicación a la especie bumana. Pris, Hermann y Cie.

Lotka, A. (1948). Applications of recurrent series in renewal theory. Annals of Mathematical Statistics, 19(2), 190-206.

Mejía de López, Á., Mora Osejo, L., y Francisco Ruiz, J. (1991). Álvaro López Toro (1926-1972). En R. Heredia y L. Mora Osejo (comps), Álvaro López Toro. Ensayos sobre Economía Demografía (pp. 17- 21). Banco de la República.

Parsons, J. (1950). La colonización antioqueña en el occidente de Colombia (E. Robledo, trad.). Imprenta Departamental de Antioquia.

Safford, F. (1967). Significación de los antioqueños en el desarrollo económico antioqueño. Universidad Nacional de Colombia. Imprenta Nacional.

Sapoznikow, J. (1976a). Un modelo macroeconómico de una economía subdesarrollada con migración interna. Documento CEDE No. 030. Universidad de los Andes.

Sapoznikow, J. (1976b). Segmentación de mercados y migración interna en países menos desarrollados. Documento CEDE No. 031. Universidad de los Andes.

Solow, R. (1956, febrero). A contribution to the theory of economic growth. Quarterly Journal of Economics, 70(1), 65-94. https://doi. org/10.2307/1884513

Swan, T. (1956, noviembre). Economic growth and capital accumulation. Economic Record, 32(2), 334-361.

Todaro, M. (1969, marzo). A model of labor migration and urban unemployment in less developed countries. American Economic Review, 59(1), 138-147. 